\title{
An Extended Hybrid Chemistry Framework for Complex Hydrocarbon Fuels
}

\author{
Rishikesh Ranade ${ }^{\mathrm{a}}$, Sultan Alqahtani ${ }^{\mathrm{a}}$, Aamir Farooq ${ }^{\mathrm{b}}$, Tarek Echekki ${ }^{\mathrm{a}}, 1$ \\ ${ }^{\mathrm{a}}$ Department of Mechanical and Aerospace Engineering, North Carolina State University, Raleigh, \\ NC 27695-7910, USA \\ ${ }^{\mathrm{b}}$ King Abdullah University of Science and Technology, Clean Combustion Research Center, Physical \\ Sciences and Engineering Division, Thuwal 23955, Saudi Arabia
}

\begin{abstract}
An extended hybrid chemistry approach for complex hydrocarbons is developed to capture hightemperature fuel chemistry beyond the pyrolysis stage. The model may be constructed based on timeresolved measurements of oxidation species beyond the pyrolysis stage. The species' temporal profiles are reconstructed through an artificial neural network (ANN) regression to directly extract their chemical reaction rate information. The $A N N$ regression is combined with a foundational $\mathrm{C}_{0}-\mathrm{C}_{2}$ chemical mechanism to model high-temperature fuel oxidation. This new approach is demonstrated for published experimental data sets of 3 fuels: $n$-heptane, $n$-dodecane and $n$-hexadecane. Further, a perturbed numerical data set for $n$-dodecane, generated using a detailed mechanism, is used to validate this approach with homogeneous chemistry calculations. The results demonstrate the performance and feasibility of the proposed approach.
\end{abstract}

Keywords: Chemistry reduction; artificial neural networks; hydrocarbon oxidation; pyrolysis.

\footnotetext{
${ }^{1}$ Corresponding Author. Address: Department of Mechanical and Aerospace Engineering, North Carolina State University, 911 Oval Drive, Campus Box 7910, Engineering Building III, Room 3252, Raleigh, NC 27695-7910, USA. Fax: +1 919515 7968, E-mail address: techekk@ncsu.edu (T. Echekki).
} 


\section{Introduction}

The oxidation chemistry of a typical transportation fuel poses severe computational challenges for multi-dimensional reacting flow simulations. These challenges may be attributed primarily to the sheer size of associated chemical mechanisms when available. However, and oftentimes, the chemical kinetic data may not be available or easily validated for a broad range of fuels. While chemistry reduction strategies have been reasonably successful in overcoming the challenge of handling chemical complexity [1], such strategies can only be used when reliable detailed mechanisms for the fuels of interest are available.

Experimental data-based chemistry reduction is one viable strategy for modeling the chemistry of complex fuels. Recently, the hybrid chemistry (HyChem) approach was proposed by Wang and coworkers [2-8] as a chemistry reduction approach for the high-temperature oxidation of transportation fuels starting from time-series measurements of fuel fragments (and other relevant species) to capture the pyrolysis stage of these fuels. Such measurements can be achieved primarily using shock tubes and a variety of optical diagnostic techniques and sampling methods. The approach is based on the premise that, at high temperatures, fuel oxidation undergoes: 1) a fast fuel pyrolysis step resulting in the formation of smaller fuel fragments, followed by 2) a longer oxidation step for these fragments, which is described with simpler $\mathrm{C}_{0}-\mathrm{C}_{4}$ foundational chemistry. While the HyChem approach enables a chemistry description, which otherwise would not be possible without the construction of detailed mechanisms for the fuel, the reliance on the $\mathrm{C}_{0}-\mathrm{C}_{4}$ foundational chemistry may be computationally prohibitive beyond the lowdimensional 0-D or 1-D computations. We have recently developed a similar framework to HyChem that is based on the two-stage oxidation process [9]. This approach has several key distinctions in its implementation compared to HyChem. It is based on a two-step regression process. In the first step, a regression is carried out on the species' concentrations time measurements using a shallow artificial neutral network (ANN). A shallow ANN uses a simple ANN architecture to generate a long-range (in 
time) regression for the time measurements to filter out the experimental noise. From the fitted temporal profiles of measured species' concentrations, rate information is obtained using the derivatives of the fitted concentrations in time. In the second step, a deep ANN regression is carried out to relate measured species' reaction rates to their concentrations. In the deep ANN, we can allow for a more complex ANN architecture in ensure a more effective regression for the reaction rates. Both the proposed framework [9] and HyChem attempt to construct reaction rate models for measured species. However, in our approach, we attempt to use the temporal data directly to construct these models. In HyChem, global reaction rate parameters and species concentration coefficients are determined as part of an optimization procedure for the global reactions.

One possible extension of the HyChem strategy is to exploit the evolution from larger (complex fuels to fragments) to smaller hydrocarbons (prescribed with $\mathrm{C}_{1}-\mathrm{C}_{2}$ chemistry) beyond the pyrolysis stage. Once the pyrolysis stage is completed, the pyrolysis fragments oxidize by evolving into even simpler hydrocarbons [10], thus, potentially enabling a simpler chemistry description than a $\mathrm{C}_{0}-\mathrm{C}_{4}$ foundational chemistry to capture the oxidation process. For experimentalists, such an extension may also require measurements of species that can be used to track the oxidation process.

In the present study, we propose and demonstrate such an extension using a recently developed framework for predicting species reaction rates based on their temporal measurements [9]. We validate our approach by using both published measurements as well as simulation data used to mimic measurements. The paper is organized as follows. First, the framework is described in Sec. 2. In Sec. 3, the framework is validated using both published experimental data and synthetic data. Finally, conclusions are presented in Sec. 4. 


\section{Principal Motivation and Implementation of the Framework}

To motivate the present approach, a constant-pressure homogenous chemistry calculation of $n$ dodecane, $n-\mathrm{C}_{12} \mathrm{H}_{26}$, is carried out using the JetSurF 2.0 [11] detailed mechanism and the Senkin code [12]. The initial conditions correspond to a stoichiometric mixture of $0.1 \% n$-dodecane/argon at a temperature of $1300 \mathrm{~K}$ and a prescribed constant pressure of $2.75 \mathrm{~atm}$. The dilution of the fuel in argon is used to emulate typical conditions used in shock tube experiments of species measurements.

(a)

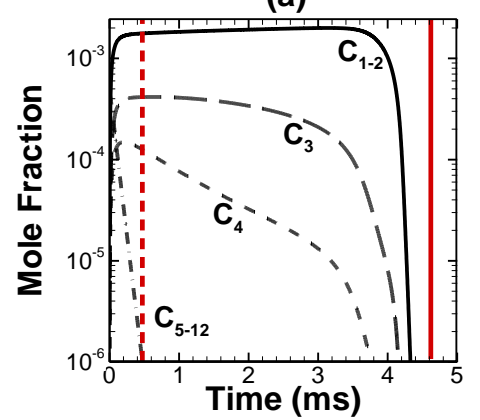

(c)

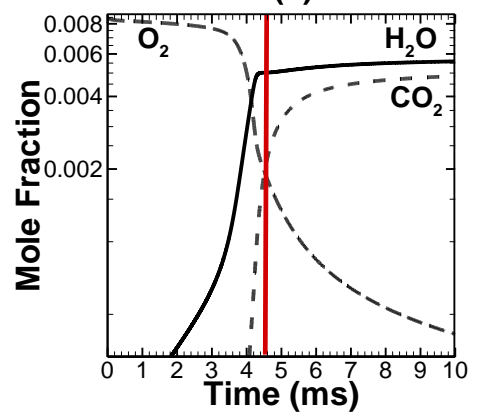

(b)

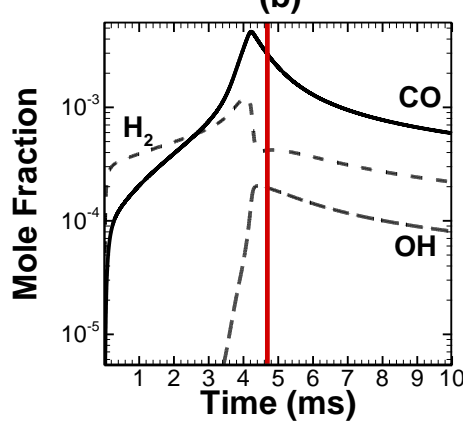

(d)

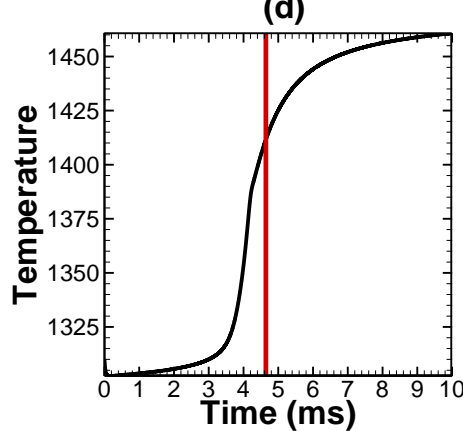

Figure 1. Detailed homogeneous chemistry calculation for stoichiometric oxidation of $n$-dodecane at a temperature of $1300 \mathrm{~K}$ and pressure of $2.75 \mathrm{~atm}$.

Figure 1 shows profiles of the temporal evolution of fragments' mole fractions, grouped by the number of carbons in their structure (Fig. 1a), key intermediates, $\mathrm{CO}, \mathrm{H}_{2}$ and $\mathrm{OH}$ (Fig. 1b), products, $\mathrm{CO}_{2}$ and $\mathrm{H}_{2} \mathrm{O}$ (Fig. 1c) and temperature (Fig. 1d). As shown in Fig. 1a, the fuel fragments with 5 or more carbon atoms break down into smaller fragments very early in the reaction; this breakdown corresponds approximately to the pyrolysis region when the HyChem global reactions for pyrolysis [2-8] are designed to operate. The end of this region is described in Fig. 1a with a dashed gray line. Next, the $\mathrm{C}_{3}$ and $\mathrm{C}_{4}$ fuel fragments are oxidized resulting in the formation of simpler molecules like $\mathrm{H}_{2}, \mathrm{CO}_{2}, \mathrm{H}_{2} \mathrm{O}$ and $\mathrm{CO}$, as shown in Figs. 1b and 1c. It may be observed that by about $4 \mathrm{~ms}$ into the reaction, the species 
corresponding to $\mathrm{C}_{0}-\mathrm{C}_{2}$ chemistry become dominant compared to the fragments of larger hydrocarbons. These species predominantly include $\mathrm{CH}_{4}, \mathrm{C}_{2} \mathrm{H}_{4}, \mathrm{H}_{2}, \mathrm{OH}, \mathrm{CO}_{2}, \mathrm{H}_{2} \mathrm{O}$ and $\mathrm{CO}$. Thus, a complex fuel like $n$ dodecane may be described in terms of $\mathrm{C}_{0}-\mathrm{C}_{2}$ chemistry after the decay of the larger fragments. We propose that the same behavior can be exploited for other large hydrocarbons, including the $n$-alkanes considered in this study.

In the approach proposed here, we use time-history measurements of these species in the region bounded by the solid gray line in Fig. 1 to build a reduced description of complex fuels. The solid gray line is chosen based on the extent of the time-history measurements of some of these species in published experimental data sets [13-15].

We adopt an ANN-based hybrid chemistry reduction methodology [9] that consists of an ANN-based model for the reactions of $\mathrm{C}_{0}-\mathrm{C}_{2}$ fuel fragments and major oxidized species like $\mathrm{H}_{2} \mathrm{O}, \mathrm{CO}_{2}, \mathrm{CO}$ and $\mathrm{OH}$, using time-history measurements, combined with a $\mathrm{C}_{0}-\mathrm{C}_{2}$ chemical mechanism for the remaining (not available from measurements) species. This methodology is based on the observation that the heavy carbon atom containing fuels break down into smaller carbon components and eventually reach a point where $\mathrm{C}_{0}-\mathrm{C}_{2}$ fuel fragments exist along with other oxidized species like $\mathrm{H}_{2} \mathrm{O}, \mathrm{CO}_{2}, \mathrm{CO}$ and $\mathrm{OH}$. The entire procedure is summarized in Fig. 2, starting with time-series measurements of oxidation species and finally resulting in a model for the reaction rates of these species.

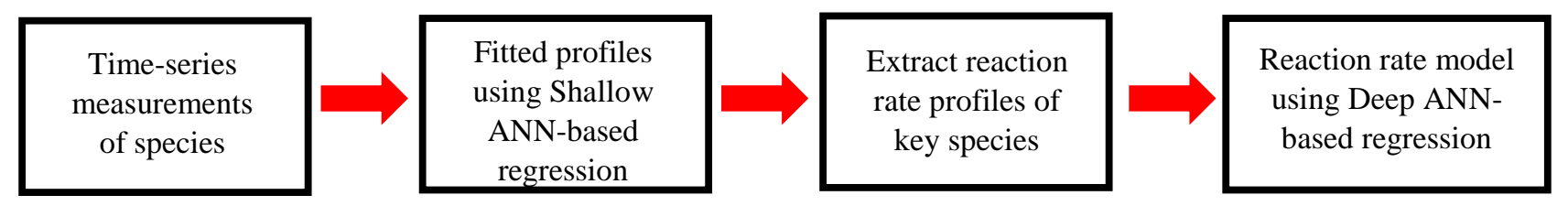

Figure 2. Schematic of the chemistry reduction process.

The ANN model is built from experimental time-history measurements of the key species using a two-step procedure as shown in Fig. 2. First, the experimental measurements of concentrations are fit using a shallow ANN to obtain predictions for smooth temporal profiles. The output of this step is a nonlinear mathematical equation relating the fitted species concentrations to time. 
An ANN architecture relates outputs to inputs through a set of layers containing a set of connections denoted as neurons. The neurons and hidden layers correspond to the complexity of the model. Further details related to the network architecture and the training algorithm may be found in our recent study [9]. The graphical form illustrated in Fig. 3 has a mathematical description that relates input and output quantities.

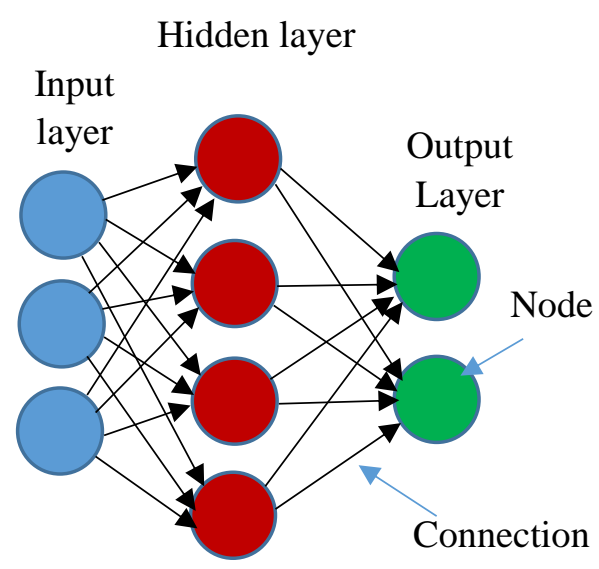

Figure 3. Schematic of an ANN with one input layer of 3 nodes, one hidden layer with 4 nodes and an output layer with 2 nodes.

For a measured time-series of a quantity $\phi$, which may correspond to species concentrations, its regression based on a single hidden layer may be expressed as:

$$
\phi(t)=W L_{\phi k} f\left(W I_{k} t+B I_{k}\right)+B L_{\phi}
$$

In this expression, $t$ is time (input); $k$ is the number of neurons in the hidden layer; $W I_{k}$ and $B I_{k}$ are the ANN weight matrix and the bias for the initial layer; $W L_{\phi k}$ and $B L_{\phi}$ are the weight matrix and the bias for the output layer; $f$ is the activation function, which corresponds, in our analysis, to the tangent sigmoid function. The weights and biases stored from the ANN training can be seen as fitting parameters for the curve fitting of time-history measurements of individual species. The number of fitting parameters used is a direct function of the number of neurons and the hidden layers. This flexibility in the shallow ANN ensures that different shapes of species profiles can be captured with ease. 
The functional form of the ANN for individual profiles can be differentiated with respect to time to determine the slopes of fitted species, resulting in an evaluation of the rates of reaction and heat release as a function of time. The resulting equation for the reaction rates $S_{\phi}$ is as follows:

$$
S_{\phi}=\frac{d \phi}{d t}=W L_{\phi k} \frac{d f}{d u_{k}} \frac{d u_{k}}{d t}, \text { where } u_{k}=W I_{k} t+B I_{k} \text { and } \frac{d u_{k}}{d t}=W I_{k}
$$

The fitted species and their corresponding rates are tabulated using a deep ANN approach. The objective of a deep ANN is to provide an accurate representation of the chemical reaction rates in terms of the transported scalars. With a more complex structure, the deep ANNs can result in an accurate model for the reaction rates that can accommodate multiple inputs, outputs and larger or more complex datasets.

In the next stage, the $\mathrm{ANN}$ based model is combined with a $\mathrm{C}_{0}-\mathrm{C}_{2}$ chemical mechanism to solve for all the species in the mechanism, as illustrated in Fig. 4. The additional reaction rates imposed by ANN model on the measured species ensure the formation of certain intermediates and radicals like $\mathrm{CO}, \mathrm{OH}$, $\mathrm{H}$, O, etc., which are handled by the $\mathrm{C}_{0}-\mathrm{C}_{2}$ mechanism along with all non-measured species. Note that, in contrast with the procedure outlined in Ref. [9], all measured species are handled entirely by a deep ANN regression model for their reaction rates due to the ability of the ANN regression to extrapolate beyond measured times in our present study. On the other hand, measured species in Ref. [9] are handled through a combination of deep ANN regression during the pyrolysis stage and a $\mathrm{C}_{0}-\mathrm{C}_{4}$ mechanism.

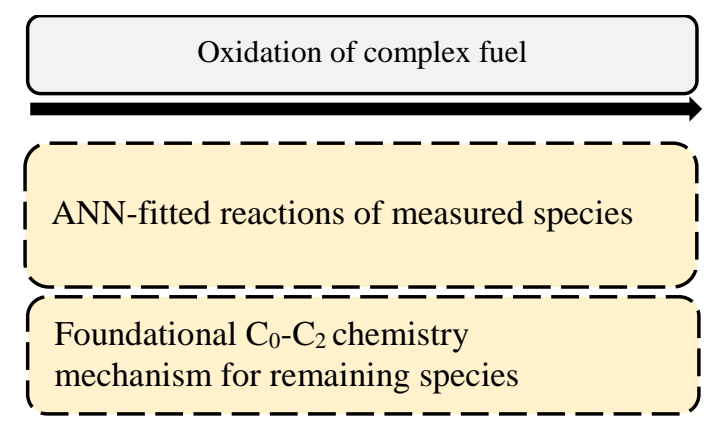

Figure 4. Reduced chemistry model for large hydrocarbon oxidation.

The proposed ANN regression approach has several advantages. With respect to time-history measurements, and as shown below, the ANN can interpolate and extrapolate data points with sufficient 
accuracy to establish the same data interval even if measurements for different species are carried out at different intervals. However, the extrapolation is only practical when the measurements are carried out sufficiently long to see the final trends of the measured quantities. Finally, in the proposed approach, ANN framework provides reaction rates for important oxidized species and certain $\mathrm{C}_{0}-\mathrm{C}_{2}$ fuel fragments, thus requiring a coupling with a simpler foundational chemistry mechanism compared to the HyChem approach [2-8].

Having presented the proposed chemistry reduction framework, it is important to emphasize the context in which this framework will be used. First, within this framework, we rely on temporal measurements of oxidation species and intermediates of the fragments' oxidation. Therefore, in contrast with the HyChem approach [2-8] or our recent work [9], the measurements must be implemented under oxidation conditions. Accordingly, the mixture stoichiometry is another parameter of the measurements, which must be considered. Second, the framework can be used for a single set of initial conditions or cover a range of conditions (e.g., equivalence ratios, temperatures and pressures) for the development of a reduced chemistry description of the high-temperature oxidation of a complex fuel. Also, as illustrated below, we can use the framework to reduce a detailed mechanism without requiring experimental measurements. The framework can combine time-series measurements (e.g., using shock tubes or rapid compression machines) with fractional yield measurements (e.g., flow reactors). Such yield measurements can be important in determining the asymptotic behavior of species when shock tube measurements are limited to a short window of pyrolysis and the initial stages of the oxidation. 


\section{Results and Discussion}

As outlined above, to fully construct a more general model for the fuel pyrolysis and oxidation, a series of time-resolved measurements of species concentrations are needed. Here, we only attempt to demonstrate the approach using prescribed initial conditions based on published experimental data sets for 3 fuels, $n$-heptane, $n$-dodecane and $n$-hexadecane [13-15], and simulated experimental profiles using $n$-dodecane homogeneous chemistry calculations. For the results presented below, the shallow ANNs for fitting species profiles use one hidden layer with 3-4 neurons, while the deep ANNs for reaction rate regression require one hidden layer and 40 neurons. The choice of neurons, hidden layers and training algorithm is similar to that in Ref. [9].

The purpose of the first portion of the study based on experimental measurements is two-fold. First, we investigate whether the shallow ANNs used to extract the measured species reaction rates can reconstruct the measured species temporal profiles. Second, we investigate if the shallow ANN regression can be used to predict the measured species trends beyond their measurement range. While the first goal is important, aspects of it have been demonstrated already in our recent study [9]. The second goal is important to establish methods to combine measurements at different time ranges or to incorporate species yield measurements at prescribed times of the temporal measurements. The ability of shallow ANN to estimate reaction rates beyond the measurement ranges is a key validation goal of this portion of the study.

In the second portion of the study, we construct simulated experimental conditions, which are obtained using detailed chemistry 0-D simulations of prescribed initial conditions and adding noise to the temporal profiles to simulate experimental conditions. Again, despite access to the temporal profiles of hundreds of species, we implement the model only with a set of species that may be measured in actual experiments. The available remaining species data provide a blind test for the model. Although, an extension of this set may be needed to build a more accurate and a more robust extended hybrid chemistry for complex fuels. 
A detailed information of the data sets associated with the first portion of this study is provided in Table 1, which summarizes fuel composition, initial temperatures, pressures and species measured with time profiles. The proposed approach is validated for each of the 3 experimental cases in Table 1 . This analysis involves the following steps: 1) a shallow ANN-regression of the temporal profiles of the measured species listed in Table 1 is first carried out; 2) the ANN regression is used to develop a common range for all measured species if the measurements involved different time intervals; 3) a regression of the chemical reaction rates for the measured species is carried out using a deep ANN; 4) the solution of a homogeneous system at constant pressure, using the prescribed conditions in Table 1, is integrated according to the extended hybrid scheme illustrated in Fig. 3. In the last step (step \#4), the ANN-based model for the measured species reaction rates is combined with a $\mathrm{C}_{0}-\mathrm{C}_{2}$ chemical mechanism. This mechanism is obtained by removing species which are more complex than $\mathrm{C}_{1}-\mathrm{C}_{2}$ hydrocarbons in the USC-Mech II mechanism [16]. This results in a foundational chemistry of 39 species and 250 elementary reactions. In contrast, the USC-Mech II [16], which has been adopted for the $\mathrm{C}_{0}-\mathrm{C}_{4}$ foundational chemistry of the HyChem approach [2-8] or our hybrid chemistry approach in [9], includes 111 species and 784 elementary reactions. Although, a more rigorous reduction strategy could have been adopted, the resulting mechanism provides a consistent subset for comparisons with the USC Mech II and the JetSurF. In addition to the adopted mechanism, we also have investigated the use of GRI Mech mechanism [17] as an alternative without seeing any noticeable differences in the results. The 0-D chemistry integration starting with a prescribed initial mixture is carried out using the Senkin code [12] at a fixed pressure.

\begin{tabular}{cccc}
\hline Fuel & Temperature (K) & Pressure (atm) & Measured Species \\
\hline $300 \mathrm{ppm} n$-heptane [13] & 1345 & 2.75 & $\mathrm{C}_{2} \mathrm{H}_{4}, \mathrm{H}_{2} \mathrm{O}, \mathrm{CO}_{2}, \mathrm{OH}$ \\
$457 \mathrm{ppm} n$-dodecane [14] & 1410 & 2.37 & $\mathrm{C}_{2} \mathrm{H}_{4}, \mathrm{H}_{2} \mathrm{O}, \mathrm{CO}_{2}, \mathrm{OH}$ \\
$1565 \mathrm{ppm} n$-hexadecane [15] & 1352 & 1.71 & $\mathrm{C}_{2} \mathrm{H}_{4}, \mathrm{H}_{2} \mathrm{O}, \mathrm{CO}_{2}, \mathrm{OH}, \mathrm{CH}_{4}$ \\
\hline
\end{tabular}

Table 1. Details of experimental data sets used.

Figures 5, 6 and 7 compare the results from the 0-D simulations based on the extended hybrid model (solid black lines) with the experimental data (solid red lines) of measured species for the 3 fuels, $n$ - 
heptane, $n$-dodecane and $n$-hexadecane, respectively. Except for $\mathrm{H}_{2} \mathrm{O}$ in Fig. 5 and $\mathrm{OH}$ in Figs. 5 - 7, the remaining species' measurements are not available to the extent of the simulations. Their chemical reaction rate regression is extrapolated beyond the measurement window using the shallow ANN.

(a)

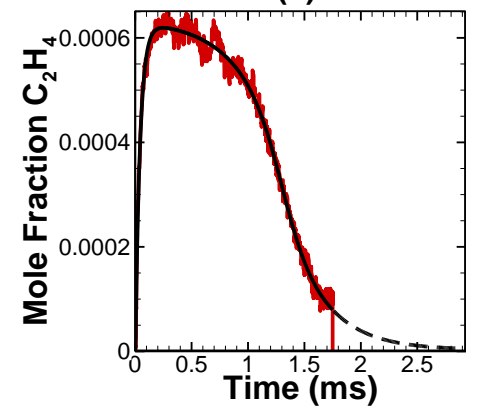

(c)

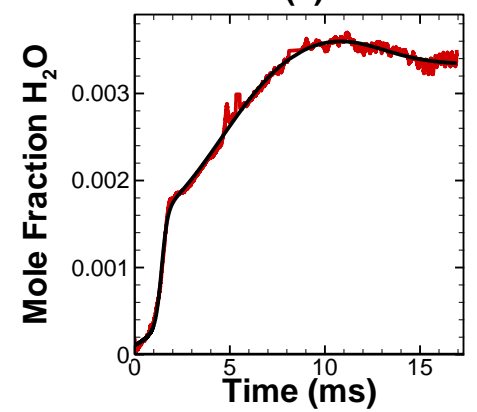

(b)

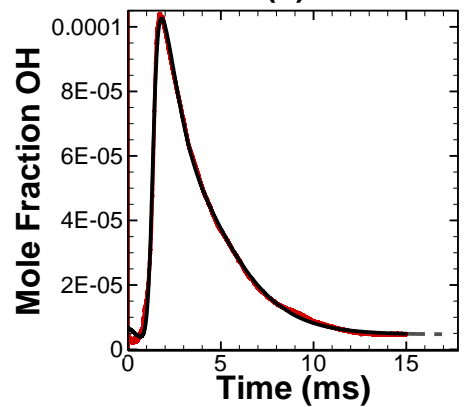

(d)

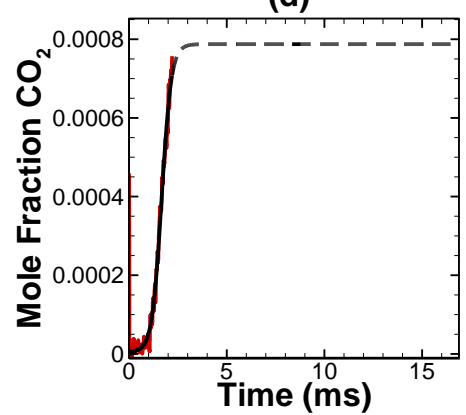

Figure 5. Validation of $n$-heptane [13] for (a) $\mathrm{C}_{2} \mathrm{H}_{4}$, (b) $\mathrm{OH}$, (c) $\mathrm{H}_{2} \mathrm{O}$ and (d) $\mathrm{CO}_{2}$. ANN fit (black) vs experimental measurements (red).

(a)

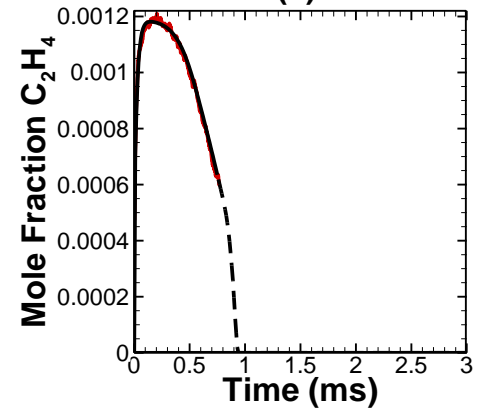

(c)

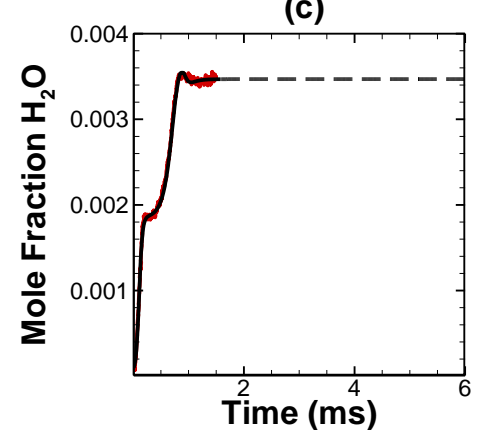

(b)

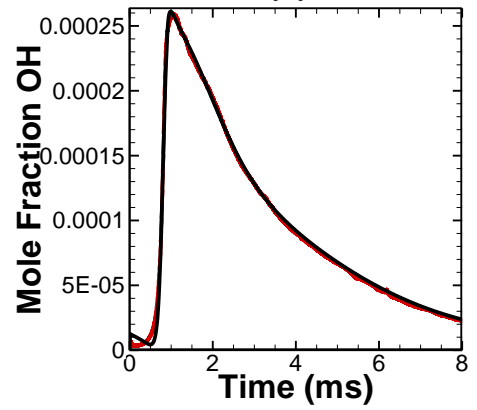

(d)

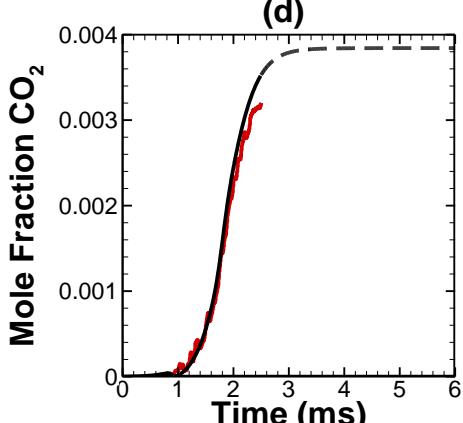

Figure 6. Validation of $n$-dodecane [14] for (a) $\mathrm{C}_{2} \mathrm{H}_{4}$, (b) $\mathrm{OH}$, (c) $\mathrm{H}_{2} \mathrm{O}$ and (d) $\mathrm{CO}_{2}$. ANN regression (black) vs experimental measurements (red). 
(a)

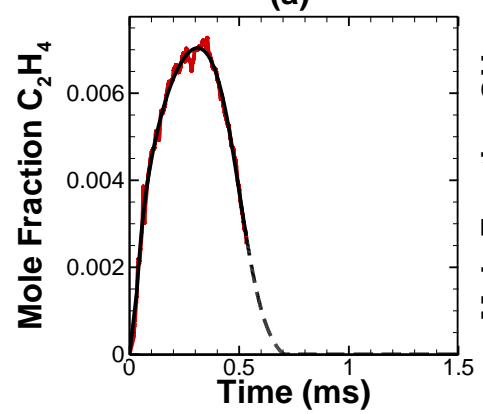

(c)

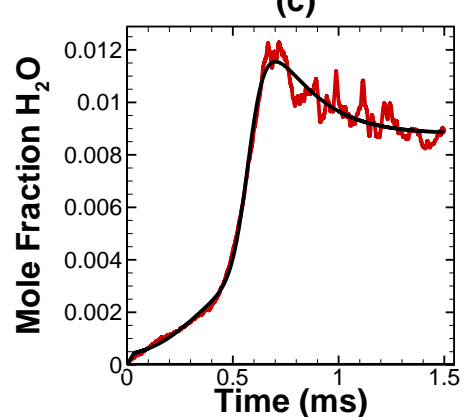

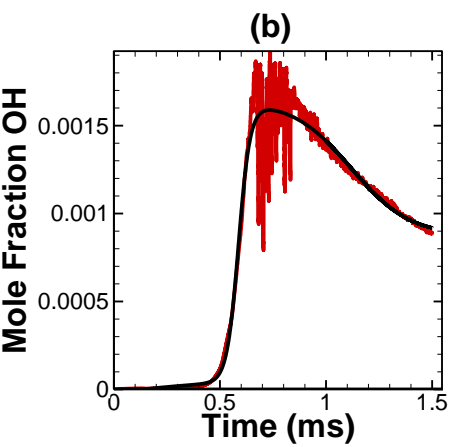

(d)

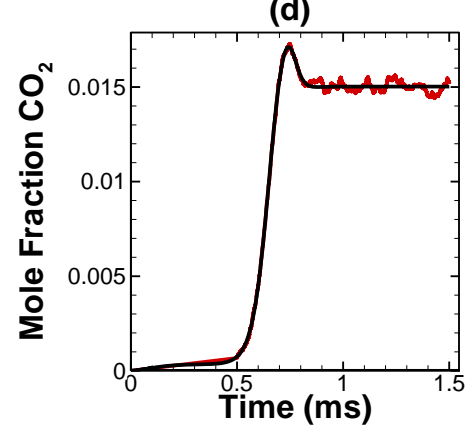

Figure 7. Validation of $n$-hexadecane [15] for (a) $\mathrm{C}_{2} \mathrm{H}_{4}$, (b) $\mathrm{OH}$, (c) $\mathrm{H}_{2} \mathrm{O}$ and (d) $\mathrm{CO}_{2}$. $\mathrm{ANN}$ regression (black) vs experimental measurements (red).

The temporal profiles match well with the experimental data in the regions modeled by the ANN (black solid). The ANN is based on the experimental data itself and hence, within the bounds of the data, it can accurately capture the time evolution of the measured species. Outside these bounds, the shallow ANN can extrapolate measured species profile reasonably well to determine species reaction rates. This ability of shallow ANN helps in handling experimental measurements with varying time intervals and different end times. The temporal profiles of all species modeled with the ANN-based chemistry behave as expected even when they are well beyond (shown as dashed lines) the extent of experimental measurements. In the presented data, $\mathrm{C}_{2} \mathrm{H}_{4}$, a fuel fragment, is consumed while the products of oxidation $\mathrm{H}_{2} \mathrm{O}$ and $\mathrm{CO}_{2}$ are seen to asymptote to their peak equilibrium values. The behavior of $\mathrm{OH}$ is also as expected. Some of the species asymptote to their equilibrium values much earlier than others depending on the initial conditions and the type of fuel. In such cases, the time ranges are adjusted to better illustrate the species trends. The species that are not a part of the experimental measurements are modeled using the $\mathrm{C}_{0}-\mathrm{C}_{2}$ chemical mechanism. 
The results presented in Figs. 5-7 only validate the model for the measured species. In the following discussion, we investigate how the proposed extended hybrid chemistry model predicts species that are not measured. For this, we generate "synthetic" measurement data by perturbing temporal profiles of species concentrations starting with a homogeneous chemistry simulation at a constant pressure. The simulation involves $n$-dodecane, $n-\mathrm{C}_{12} \mathrm{H}_{26}$, as a fuel and the detailed kinetic mechanism JetSurF 2.0 [11] is used to validate the species of $\mathrm{C}_{0}-\mathrm{C}_{2}$ chemical mechanism which are not measured by the synthetic experiment. The JetSurF 2.0 [11] mechanism consists of 348 species and 2163 reactions. Numerical simulations are carried out using the Senkin code [12] for a stoichiometric mixture of $0.1 \% n$ dodecane/argon at a pressure of $2.75 \mathrm{~atm}$ and an initial temperature of $1300 \mathrm{~K}$, which is the same condition presented in Fig. 1. Species measured in experiments [13-15] described previously are extracted from this numerical simulation. To emulate experimental data, noise is added to the temporal profiles of the simulated species, $\mathrm{C}_{2} \mathrm{H}_{4}, \mathrm{H}_{2} \mathrm{O}, \mathrm{CO}_{2}$ and $\mathrm{OH}$. This noise is modeled by adding fluctuations from a uniform random distribution with a magnitude of $10 \%$ of the maximum concentration of the measured quantities. For a measured specie, $\phi$, this perturbation can be expressed as:

$$
\phi_{\text {perturbed }}(t)=\phi_{\text {simulated }}(t)+0.1 \times r \times \phi_{\max }
$$

where $r$ is a random function ranging from -1 to 1 . Therefore, the relative error established by this model can be very large for the measured species when their concentrations are low. A $10 \%$ error is chosen in this case as opposed to $30 \%$ in Ref. [9] because the species that are tabulated here are all major products of oxidation and tend to have relatively large peak concentrations, more than 100 times as that of the pyrolysis fragments. Nonetheless, as discussed in Ref. [9], a higher error may be accommodated by carrying out the temporal measurements more than once. It is important to note that, while the extended chemistry model is validated for a list of 4 "measured" species, we expect the model to be more accurate if additional species are measured. This fact will be demonstrated while discussing the results below.

Next, the methodology described in Figs. 2 and 4 is implemented on the perturbed simulation data. Here, the extended hybrid model includes ANN regression for the chemical reaction rates for $\mathrm{C}_{2} \mathrm{H}_{4}, \mathrm{H}_{2} \mathrm{O}$, 
$\mathrm{CO}_{2}$ and $\mathrm{OH}$ (the deep ANN outputs), which are considered to be the only species measured by the experiment. Therefore, the ability of the extended hybrid scheme to predict other $\mathrm{C}_{0}-\mathrm{C}_{2}$ species, which are not part of the 4 selected species is an important validation step of the scheme. Finally, ANN regression for the measured species reaction rates can in principle include all measured species concentrations as inputs or only a partial list. This is a flexibility offered by ANN regression, and this may be convenient under conditions where either one or more of the measured species is strongly correlated with the rest of the measured species or its measurement has a relatively low signal-to-noise ratio. In the present study, we have found that the measured species reaction rates (the outputs to the deep ANN) can be adequately described with only $\mathrm{C}_{2} \mathrm{H}_{4}, \mathrm{CO}_{2}$ and $\mathrm{H}_{2} \mathrm{O}$ concentrations as inputs in the deep ANN (i.e., $\mathrm{OH}$ can be omitted as an input to the deep ANN without altering the results).

(a)

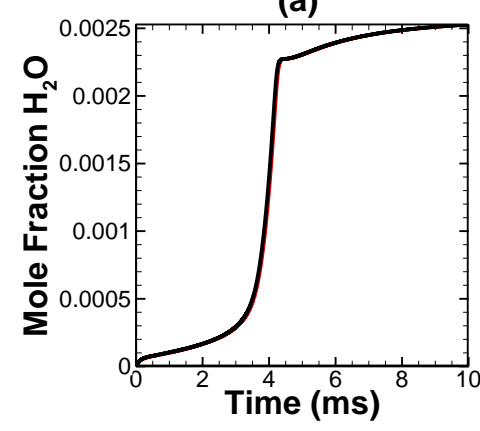

(c)

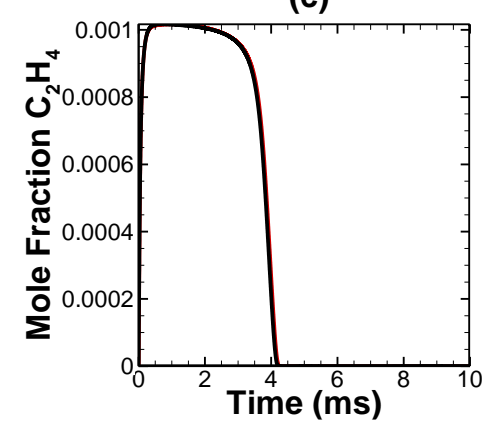

(b)

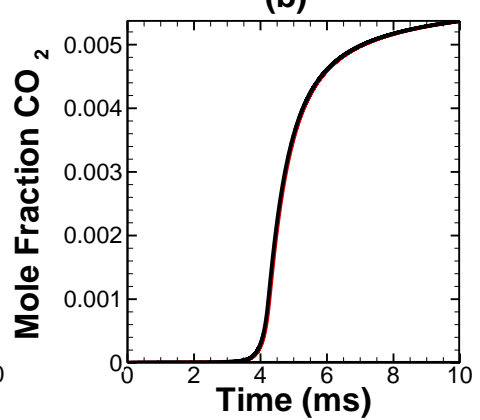

(d)

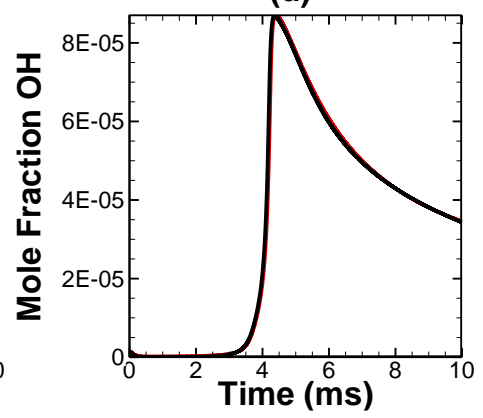

Figure 8. Comparison of species mole fractions temporal profiles based on: the extended hybrid chemistry scheme (black) and the detailed mechanism simulations (red) for the "measured" species: (a) $\mathrm{H}_{2} \mathrm{O}$, (b) $\mathrm{CO}_{2}$, (c) $\mathrm{C}_{2} \mathrm{H}_{4}$ and (d) $\mathrm{OH}$.

Figure 8 compares the evolution of species $\mathrm{H}_{2} \mathrm{O}, \mathrm{CO}_{2}, \mathrm{C}_{2} \mathrm{H}_{4}$ and $\mathrm{OH}$ based on the extended hybrid scheme (solid lines) as well as detailed chemistry calculation using JetSurF 2.0 (dashed lines). These species are modeled by ANN and are expected to agree with the detailed chemistry calculations. The real 
test of this scheme will depend upon the comparisons of species' profiles which are completely handled by the $\mathrm{C}_{0}-\mathrm{C}_{2}$ chemistry mechanism and are not part of the measurement data.

Figure 9 compares the evolutions of a reaction progress variable, $\mathrm{O}_{2}$, and three key intermediates of the oxidation process, $\mathrm{CO}, \mathrm{H}_{2}$ and $\mathrm{H}$, based on the extended hybrid chemistry scheme (solid lines) with the detailed chemistry calculations (dashed lines). Neither of the species shown are part of the measurements; therefore, these are modeled entirely with the $\mathrm{C}_{0}-\mathrm{C}_{2}$ chemistry model and their modeling is based on the limited measurements of 4 species (Fig. 8). Due to the simplicity of this model, certain reactions contributing to the production and consumption of these species may not be captured and, as a result, some deviations in the species' profiles can be observed initially. An obvious example is the deviation of the $\mathrm{H}_{2}$ mass fraction from the detailed chemistry calculations in Fig. 9. Nonetheless, the list of only 4 measured species results in a reasonable approximation of the mixture evolution.

(a)

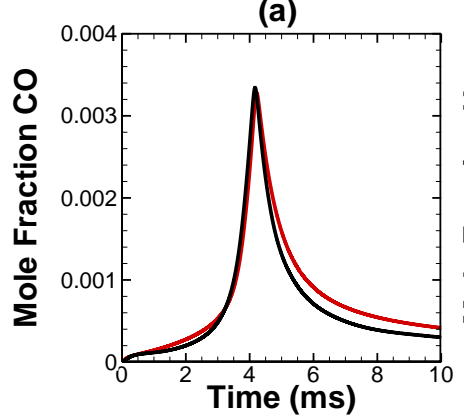

(c)

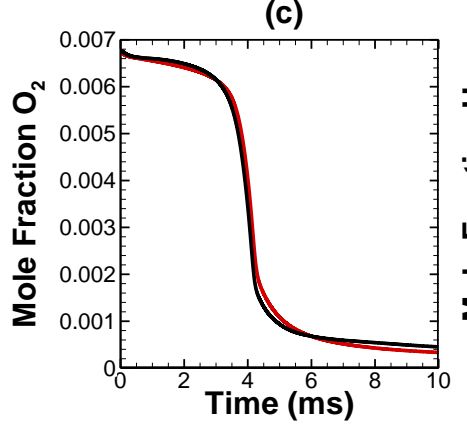

(b)

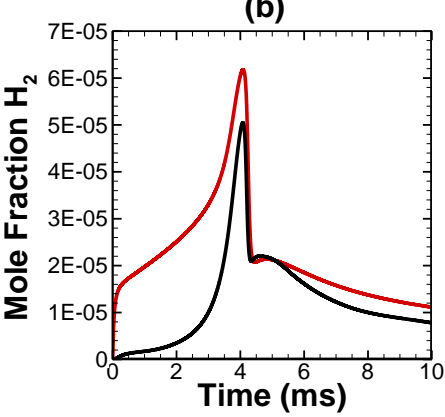

(d)

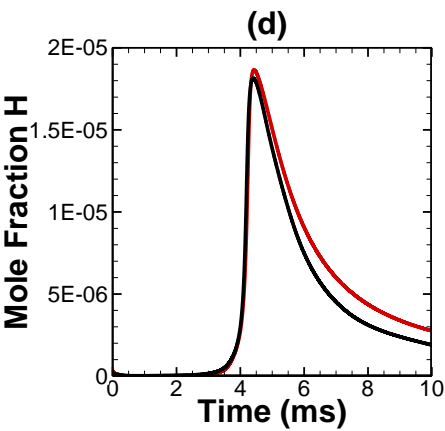

Figure 9. Comparison of non-measured species mole fractions temporal profiles using based on: the extended hybrid chemistry scheme (black) and the detailed mechanism simulations (red) for the "non-measured" species: (a) $\mathrm{CO}$, (b) $\mathrm{H}_{2}$, (c) $\mathrm{O}_{2}$ and $(\mathrm{d}) \mathrm{H}$.

To illustrate the sensitivity of the model to the omission of major species measurements, we add $\mathrm{O}_{2}$ and $\mathrm{H}_{2}$ to the list of measured species and carry out the same procedure as above. Since $\mathrm{O}_{2}$ and $\mathrm{H}_{2}$ are symmetric molecules and do not have rotational or vibrational frequencies with infrared/microwave laser 
light, they may need to be measured with sampling-based methodologies. To account for this, we consider a much higher percentage error of $30 \%$ in the time-history measurements of $\mathrm{O}_{2}$ and $\mathrm{H}_{2}$ as opposed to $10 \%$ for the rest of the measured species. The same species shown in Fig. 9 are reproduced in Fig. 10 with $\mathrm{C}_{2} \mathrm{H}_{4}, \mathrm{H}_{2} \mathrm{O}, \mathrm{CO}_{2}, \mathrm{OH}, \mathrm{O}_{2}$ and $\mathrm{H}_{2}$ as measured species. The figure shows a significant improvement in the prediction of all species compared to Fig. 9. Although not shown here, including minor species or species with much lower concentrations than $\mathrm{O}_{2}$ and $\mathrm{H}_{2}$ in the measured list has a marginal effect on the prediction of species evolutions beyond what is shown in Fig. 10. Any minor deviations in the profiles of $\mathrm{O}_{2}$ and $\mathrm{H}_{2}$ in Fig. 10 may be attributed to the large error induced in their time-history measurements. As outlined in our earlier study [9], the prediction of measured species profiles and their reaction rates can be improved by repeating the time-series measurements and combining the different experimental profiles that are fed to the shallow ANN.

(a)

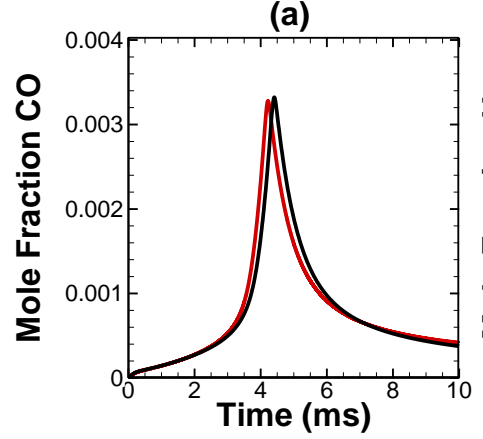

(c)

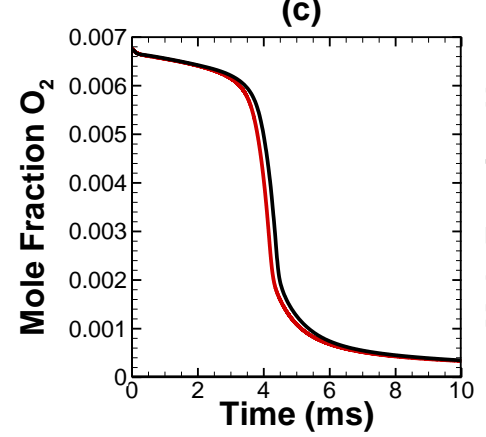

(b)

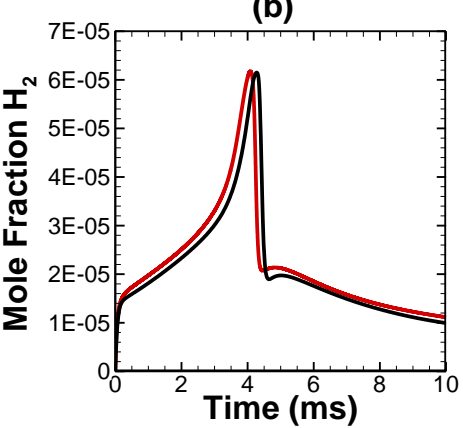

(d)

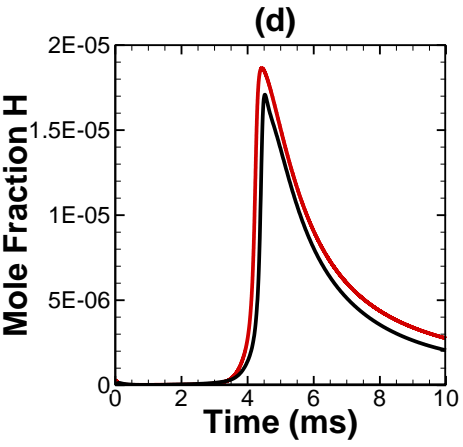

Figure 10. Comparison of species mole fractions temporal profiles based on: the extended hybrid chemistry scheme (black) and the detailed mechanism simulations (red) for the species: (a) CO, (b) $\mathrm{H}_{2}$, (c) $\mathrm{O}_{2}$ and (d) $\mathrm{H}$. The measured species include $\mathrm{H}_{2} \mathrm{O}, \mathrm{CO}_{2}, \mathrm{C}_{2} \mathrm{H}_{4}, \mathrm{OH}, \mathrm{O}_{2}$ and $\mathrm{H}_{2}$.

At this stage, it is important to comment on the potential computational saving of adopting an extended hybrid chemistry approach as presented here, compared to the hybrid chemistry approach based on 
pyrolysis and coupled with $\mathrm{C}_{0}-\mathrm{C}_{4}$ chemistry [2-9] or based on detailed chemistry. In our present study, this saving is primarily associated with the foundational chemistry. This chemistry requires about one third of the species and chemical reactions as the $\mathrm{C}_{0}-\mathrm{C}_{4}$ chemistry, resulting in about one third reduction in the computational time. An approximately one order of magnitude reduction in computational time is achieved with the extended hybrid chemistry scheme compared to the use of detailed mechanism based on JetSurF 2.0 [11]. In multi-dimensional CFD simulations, the additional savings in memory can be critical to model realistic engine geometries.

Finally, having established the feasibility of chemistry reduction with fuel fragments and major oxidation products, chemistry reduction over a range of equivalence ratios, initial temperatures and pressures for a given fuel can be developed based on parametric time-series measurements. The general strategy is already outlined in Ref. [9]. Adaptive deep ANN tabulations, to accommodate similar states in composition space and to optimize ANN, can be achieved using self-organizing maps (SOMs) clustering. Such maps have been explored in the past within the context of chemistry tabulation by Blasco et al. [18] and Franke et al. [19]. Moreover, the clustering enables the construction of 'local' models on individual datasets instead of a single 'global' model, thus enabling the framework to handle very large datasets without increasing the size of the model. Additionally, clustering may enable the use of low-order polynomial regressions instead of the use of deep ANNs to optimize the computational performance of chemistry tabulation.

The proposed ANN-based chemistry reduction framework also can be applied to existing chemical mechanism as a method of chemistry reduction for high-temperature combustion of complex fuels. A reduction from a complex fuel mechanism of thousands or tens of thousands of reactions to a simpler $\mathrm{C}_{0^{-}}$ $\mathrm{C}_{2}$ model can be further coupled with standard chemistry reduction methods to derive a computationally affordable mechanism for complex fuels. 


\section{Conclusions}

An ANN-based regression/tabulation framework is proposed to derive an extended hybrid chemistry scheme for the high-temperature oxidation of large hydrocarbons starting with experimental time series data of key oxidized products and fuel fragments. The framework is based on the premise that for most hydrocarbons, the evolution of the fuel from pyrolysis to the formation of pyrolysis fragments proceeds from complex to simpler hydrocarbons to eventually evolve into a chemical system that can be handled by a $\mathrm{C}_{0}-\mathrm{C}_{2}$ mechanism. This work extends the HyChem paradigm [2-8] to involve measurements of key oxidation species, resulting in a coupling with simpler foundational chemistry.

The proposed framework is validated using published experimental measurements of 3 fuels and numerically perturbed data. The results show that the proposed approach of ANN-based extended hybrid chemistry can yield effective and accurate chemistry reduction scheme for heavy hydrocarbon fuels and thus provide a significant improvement in terms of chemistry reduction.

With the availability of experimental data at a broader range of conditions for pressure, temperature, equivalence ratio and fuel dilution, this approach can potentially be extended to building a full-scale reduced mechanism for heavy hydrocarbon fuels. It also can be used to fit only one set of experimental conditions and can be implemented as a chemistry reduction scheme for detailed mechanisms.

Finally, while we find the shallow ANN to be a useful regression method to fit the measured species' concentrations temporal profiles, there is probably a range of regression methods that can be adopted for the measured species' reaction rates as alternatives to the deep ANN. We will be exploring different regression methods in the future that will address both computational cost and accuracy considerations.

\section{Acknowledgements}

Dr. Aamir Farooq would like to thank the Office of Sponsored Research at the King Abdullah University of Science and Technology (KAUST) for financial support. Sultan Alqahtani would like to acknowledge the support of King Khalid University in Abha, Saudi Arabia. 


\section{References}

1. T. Turanyi, A.S. Tomlin, Reduction of reaction mechanisms, in Analysis of Kinetic Reaction Mechanisms, pp. 183-312, Springer, 2014.

2. R. Xu, H. Wang, D.F. Davidson, R.K. Hanson, C.T. Bowman, F.N. Egolfopoulos, Evidence supporting a simplified approach to modeling high-temperature combustion chemistry, $10^{\text {th }}$ U.S. National Combustion Institute Meeting, The Combustion Institute, College Park, MD, April 23-26, 2017.

3. R. Xu, D. Chen, K. Wang, T. Tao, J.K. Shao, T. Parise, Y. Zhu, S. Wang, R. Zhao, D.J. Lee, F.N. Egolfopoulos, D.F. Davidson, R.K. Hanson, C.T. Bowman, H. Wang, HyChem Model: Application to petroleum-derived jet fuels, $10^{\text {th }}$ U.S. National Combustion Institute Meeting, The Combustion Institute, College Park, MD, April 23-26, 2017.

4. K. Wang, R. Xu, T. Parise, J.K. Shao, D.J. Lee, A. Movaghar, D.F. Davidson, R.K. Hanson, H. Wang, C.T. Bowman, F.N. Egolfopoulos, Combustion kinetics of conventional and alternative jet fuels using a hybrid chemistry (HyChem) approach, 10 $1{ }^{\text {th }}$ U.S. National Combustion Institute Meeting, The Combustion Institute, College Park, MD, April 23-26, 2017.

5. K. Wang, R. Xu, T. Parise, J.K. Shao, D.F. Davidson, R.K. Hanson, H. Wang, C.T. Bowman, Evaluation of a hybrid chemistry approach for combustion blended petroleum and bio-derived jet fuels, $10^{\text {th }}$ U.S. National Combustion Institute Meeting, The Combustion Institute, College Park, MD, April 23-26, 2017.

6. R. Xu, D. Chen, K. Wang, H. Wang, A comparative study of combustion chemistry of conventional and alternative jet fuels with hybrid chemistry approach, $55^{\text {th }}$ AIAA Aerospace Sciences Meeting, AIAA paper 2017-0607, Grapevine, Texas, January 9-13, 2017.

7. H. Wang, R. Xu, K. Wang, C.T. Bowman, R.K. Hanson, D.F. Davidson, K. Brezinsky, F.N. Egolfopoulos, A physics-based approach to modeling real-fuel combustion chemistry-I. Evidence 
from experiments, and thermodynamic, chemical kinetic and statistical considerations. Combust. Flame 193 (2018) 502-519.

8. R. Xu, K. Wang, S. Banerjee, J.K. Shao, T. Parise, Y.Y. Zhu, S.K. Wang, A. Movaghar, D.J. Lee, R.H. Zhao, X. Han, Y. Gao, T.F. Lu, K. Brezinsky, F.N. Egolfopoulos, D.F. Davidson, R.K. Hanson, C.T. Bowman, H. Wang, A Physics-based approach to modeling real-fuel combustion chemistry-II. Reaction kinetic models of jet and rocket fuels, Combust. Flame 193 (2018) 520-537.

9. R. Ranade, S. Alqahtani, A. Farooq, T. Echekki, An ANN based hybrid chemistry framework for complex fuels, Fuel 241 (2019) 625-636.

10. C.K. Westbrook, F.L. Dryer, Chemical kinetics modeling of hydrocarbon combustion, Prog. Energy Combust. Sci. 10 (1984) 1-57.

11. H. Wang, E. Dames, B. Sirjean, D. A. Sheen, R. Tango, A. Violi, J. Y. W. Lai, F. N. Egolfopoulos, D. F. Davidson, R. K. Hanson, C. T. Bowman, C. K. Law, W. Tsang, N. P. Cernansky, D. L. Miller, R. P. Lindstedt, A high-temperature chemical kinetic model of $n$-alkane (up to $n$-dodecane), cyclohexane, and methyl-, ethyl-, n-propyl and n-butyl-cyclohexane oxidation at high temperatures, JetSurF version 2.0, September 19, 2010 (http://web.stanford.edu/group/haiwanglab/JetSurF/JetSurF2.0/index.html).

12. A.E. Lutz, R.J. Kee, J.A. Miller. SENKIN: A FORTRAN program for predicting homogeneous gas phase chemical kinetics with sensitivity analysis. No. SAND-87-8248. Sandia National Labs. Livermore, CA (USA), 1988.

13. D.F. Davidson, Z. Hong, G.L. Pilla, A. Farooq, R.D. Cook, R.K. Hanson, Multi-species time-history measurements during $n$-heptane oxidation behind reflected shock waves, Combust. Flame 157 (2010) 1899-1905.

14. D. F. Davidson, Z. Hong, G. Pilla, A. Farooq, R. Cook, R. K. Hanson, Multi-species time-history measurements during $n$-dodecane oxidation behind reflected shock waves, Proc. Combust. Inst. 33 (2011) 151-157. 
15. D. Haylett, D. F. Davidson, R. Cook, Z. Hong, W. Ren, S. Pyun, R. K. Hanson, Multi-species time history measurements during $n$-hexadecane oxidation behind reflected shock waves, Proc. Combust. Inst. 34 (2013) 369-376.

16. H. Wang, X. You, A.V. Joshi, S.G. Davis, A. Laskin, F. Egolfopoulos, C.K. Law, USC Mech Version II. High-Temperature Combustion Reaction Model of $\mathrm{H}_{2} / \mathrm{CO} / \mathrm{C}_{1}-\mathrm{C}_{4}$ Compounds. http://ignis.usc.edu/USC_Mech_II.htm, May 2007.

17. G. P. Smith, D. M. Golden, M. Frenklach, N.W. Moriarty, B. Eiteneer, M. Goldenberg, C.T. Bowman, R.K. Hanson, S. Song, W.C. Gardiner, V.V. Lissianski, Z. Qin, GRI-Mech 3.0 (http://www.me.berkeley.edu/gri_mech/).

18. J.A. Blasco, N. Fueyo, C. Dopazo, J.Y. Chen, A self-organizing-map approach to chemistry representation in combustion applications, Combust. Theo. Model. (2000) 61-76.

19. L.L.C. Franke, A.K. Chatzopoulos, S. Rigopoulos, Tabulation of combustion chemistry via artificial neural networks: Methodology and application to LES-PDF simulation of Sydney flame L, Combust. Flame 185 (2017) 245-260. 